\title{
The Influence of Gastrodin on Expression of IL-10, STAT3 and JAK2 in Epileptic Rats' Hippocampus
}

\author{
Xian Li, Xianglin Cheng* \\ Department of Neurology, The Clinical Medicine School of Yangtze University, The First Affiliated Hospital of Yangtze \\ University, Jingzhou, China \\ Email: ^45423626@qq.com
}

How to cite this paper: $\mathrm{Li}, \mathrm{X}$. and Cheng, X.L. (2018) The Influence of Gastrodin on Expression of IL-10, STAT3 and JAK2 in Epileptic Rats' Hippocampus. Yangtze Medicine, 2, 18-27.

https://doi.org/10.4236/ym.2018.21002

Received: November 28, 2017

Accepted: March 24, 2018

Published: March 27, 2018

Copyright (๑) 2018 by authors and Scientific Research Publishing Inc. This work is licensed under the Creative Commons Attribution International License (CC BY 4.0).

http://creativecommons.org/licenses/by/4.0/ (c) (i) Open Access

\begin{abstract}
Objective: To explore the influence of gastrodin on IL-10, JAK2 and STAT3 in hippocampus of epileptic rats induced by pentylenetetrazol and the role of the IL-10 pathway in epilepsy. Methods: 50 adult male Wistar rats were randomly divided into 5 groups: normal control group (NC Group), epilepsy model group (EP Group), low doses of gastrodin + EP Group (GE1 Group), medium doses of gastrodin + EP Group (GE2 Group), high doses of gastrodin + EP Group (GE3 Group). EP group and GE Groups were injected subthreshold doses of pentylenetetrazole (PTZ) by intraperitoneal once a day until reaching the ignited standards. GE groups were respectively injected $4,6,8 \mathrm{mg} / \mathrm{kg}$ gastrodin by intraperitoneal. All groups were administered for 28 consecutive days. The behavioral changes of the rats were observed and recorded daily 1 hour after the injection. mRNAs of IL-10, STAT3 and JAK2 in hippocampus were measured by RT-qPCR, and proteins by Western blot. Results: Compared GE2 group with EP group, the incubation of seizure was significantly prolonged $(\mathrm{P}<0.01)$, the duration was shorter $(\mathrm{P}<0.01)$ and the intensity was reduced $(\mathrm{P}<0.05)$. Compared with NC group, IL-10 expression levels in hippocampus were significantly decreased in EP group, and JAK2 and STAT3 expression levels were significantly increased. Compared with EP group, IL-10 expression levels in hippocampus were significantly increased in GE2 group, and STAT3 and JAK2 expression levels were significantly decreased in GE2 groups. The mRNA and protein levels of STAT3 and JAK2 were higher while IL-10 was lower in the PTZ group than in the NC group. However, the mRNA and protein levels of STAT3 and JAK2 were lower while IL-10 was higher in the GE2 group than in the PTZ group. Conclusions: Gastrodin can increase the expression of IL-10, and reduce the expression of STAT3 and JAK2, which may play an antiepileptic effect through regulating JAK2/STAT3 signaling pathways by IL- 10 .
\end{abstract}




\section{Keywords}

Gastrodin, Epilepsy, IL-10, STAT3/JAK2 Signal Pathway

\section{Introduction}

Epilepsy is a chronic, repetitive, paroxysmal self-limiting syndrome. It's caused by various brain diseases, characterized the neuron highly synchronized abnormal discharge. There are $600-1000$ million epilepsy patients in China and 65 70 million new cases every year [1]. Inflammation is one of the key factors in the occurrence and seizure of epilepsy [2]. On the other hand, cytokines are the most basic substances of regulation inflammation and play a key role in the development of epilepsy [3].

Interleukin-10(IL-10) is a recently discovered immunosuppressive factor with multiple bioactivity. It plays an important role in inflammation in the development of various diseases such as intestinal inflammation, chronic infection, tumor surveillance and neurodegenerative diseases [4] [5] [6] [7]. In the central nervous system, IL-10 is not only an important immune regulatory factor, but also an important neurotransmitter, which participates in the pathogenesis of epilepsy through various ways. Researching the role of IL-10 in the progress of epilepsy is very important to elucidate the pathogenesis of epilepsy and to prevent epilepsy. Gastrodin is an active ingredient extracted from traditional Chinese medicinal herbs gastrodia elata. We have found that gastrodin has an antagonistic effect on epilepsy in early animal experiment and clinical application [8], but it is necessary to further study and clarify its specific mechanism. Therefore, we study the effects of gastrodin on the expression of IL-10, Jak2 and STAT3 in the inflammatory signaling pathway of hippocampal neurons on the basis of the preliminary study, to explore the mechanism of gastrodin in the treatment of epilepsy, and lay a theoretical foundation for the further development and utilization of gastrodin.

\section{Materials and Methods}

\subsection{Animals and Reagents}

The subject was approved by the Medical Ethics Committee of the first Clinical Medical College of Yangtze University, and the animal model was completed in the Animal Experiment Center of Hubei Traditional Chinese Medicine College.

Fifty 7 - 8-week-old healthy male Wistar rats, weight $120 \pm 15$ g, provided by the Three Gorges University Laboratory Animal Center, License No.: SCXK (E): 2011-0012; natural day and night, ambient temperature $22^{\circ} \mathrm{C}-25^{\circ} \mathrm{C}$, feeding in cages and free to eat.

PTZ (CAS: 54-95-5) from Sigma; gastrodin from Kunming Pharmaceutical Group Co., Ltd. (Lot: Sinopharm H20013046); Rabbit monoclonal antibody 
JAK2 (CAS: 3230) and STAT3 (CAS: 4904T) from Cell Signaling; Rat monoclonal IL-10 antibody (CAS: ab33471) from Abcam; Rabbit polyclonal antibody $\beta$-actin (CAS: ANT010) and HRP mark Rabbit anti-rat Ig-G (CAS: ANT022) from Antgene; HRP mark Goat anti-rabbit Ig-G (CAS: GTX213110) from Genetex company; Reverse transcriptase Kit and RT-PCR kit from Beyotime; JAK2, IL-10, STAT3 and $\beta$-actin primers from Takara; electrophoresis instrument provided by Bio-rad; RT-PCR instrument ABI7500 provided by Thermo Fisher.

\subsection{Group and Treatment}

The rats were randomly divided into 5 groups, 10 per group: normal control group (NC), epilepsy model group (EP), low doses gastrodin + EP Group (GE1), medium doses gastrodin + EP Group (GE2), high doses gastrodin + EP Group (GE3). The dosage of gastrodin was considered as adult. PTZ was prepared into $10 \mathrm{mg} / \mathrm{ml}$ (1\%) with normal saline (NS) before using, gastrodin $2 \mathrm{mg} / \mathrm{ml}$, stored at $4^{\circ} \mathrm{C}$. Treated the rats at 15:00 - 17:00, observed them $1 \mathrm{~h}$ after the treatment, recorded the seizure incubation, duration and intensity of each group. The treatment was intraperitoneal injection with a duration of 28 days. The doses used as in Table 1.

\subsection{Behavioral Evaluation}

We measured the incubation from PTZ injection to myoclonic jerks, the duration from myoclonic jerks to seizures ending. The behavioral changes were recorded for $1 \mathrm{~h}(\mathrm{n}=10)$. The seizure stage was evaluated based on the Racine grading standard [9]: Stage 0: no reaction; Stage 1: wet dog shiver, facial muscle twitch, chew; Stage 2: rhythmic nodding; Stage 3: paroxysmal spasm of one forelimb; Stage 4: when standing, paroxysmal spasm and rigidity of both forelimbs; Stage 5: lost balance, dumping, limbs twitching, whole body clonus. The chronic ignition standard is reached when 5 consecutive attack of grade II above.

\subsection{Detect Hippocampal IL-10, STAT3 and JAK2 Gene Expressing by RT-qPCR}

In the 28th day, we randomly selected 4 rats in each group, $20 \mathrm{mg}$ hippocampus for RT-qPCR, corresponding to the same side of the remaining hippocampus for Western blot. The total RNA was extracted and purified by the instruction manual, determined by ultra-micro-ultraviolet spectrophotometer, detected the

Table 1. The treatment of the rats.

\begin{tabular}{ccc}
\hline Group & $15: 00-16: 00$ & $16: 00-17: 00$ \\
\hline NC & NS, $35 \mathrm{mg} / \mathrm{kg}$ & $\mathrm{NS}, 6 \mathrm{mg} / \mathrm{kg}$ \\
EP & PTZ, $35 \mathrm{mg} / \mathrm{kg}$ & $\mathrm{NS}, 6 \mathrm{mg} / \mathrm{kg}$ \\
GE1 & $\mathrm{PTZ}, 35 \mathrm{mg} / \mathrm{kg}$ & Gastrodin, $4 \mathrm{mg} / \mathrm{kg}$ \\
GE2 & PTZ, $35 \mathrm{mg} / \mathrm{kg}$ & Gastrodin, $6 \mathrm{mg} / \mathrm{kg}$ \\
GE3 & PTZ, $35 \mathrm{mg} / \mathrm{kg}$ & Gastrodin, $8 \mathrm{mg} / \mathrm{kg}$ \\
\hline
\end{tabular}


integrity of RNA by denatured agarose gel electrophoresis, reverse transcription synthesis of cDNA, IL-10 JAK2 and STAT3 mRNA expression in the hippocampus were detected by RT-PCR kit. The sequences of each gene primer are as in Table 2.

The total reaction volume is $25 \mathrm{ul}$. qPCR reaction conditions as follows: $95^{\circ} \mathrm{C}$, $2 \mathrm{~min} \rightarrow\left(95^{\circ} \mathrm{C} 15 \mathrm{~s}, 60^{\circ} \mathrm{C} 30 \mathrm{~s}\right) \times 40$ cycles. Finally do dissolution curve analysis $\left(95^{\circ} \mathrm{C} 15 \mathrm{~s}, 60^{\circ} \mathrm{C} 15 \mathrm{~s}, 95^{\circ} \mathrm{C} 15 \mathrm{~s}\right)$. The relative quantitative analysis was carried out by $2-\Delta \Delta \mathrm{Ct}$ method [10]: the expression of related genes was calculated by the difference $(\Delta \mathrm{Ct})$ of $\beta$-actin RNA cyclic threshold $(\mathrm{Ct})$. Calculate the differences in gene expression between the contrastive samples $(\Delta \Delta \mathrm{Ct})$, relative quantitation of multiple of expression differences was $2-\Delta \Delta \mathrm{Ct}$, the relative quantitation value of normal group is 1 , the $2-\Delta \Delta \mathrm{Ct}$ value of each group is a change multiple relative to 1 .

\subsection{Detect Hippocampal IL-10, STAT3 and JAK2 Protein Expressing by Western Blot}

Extracted the total protein of hippocampus with conventional method, quantified according to BCA method. Took 20 ul protein solution, added 5 ul $5 \times$ SDS-PAGE sample loading buffer, $100^{\circ} \mathrm{C}$ degeneration $5 \mathrm{~min}$, took $30 \mathrm{ug} / \mathrm{holes}$, $10 \%$ SDS-PAGE electrophoresis vertical and transferred onto PVDF membrane, blocked for $1 \mathrm{~h}$ with $5 \%$ skim milk (TBST preparation) at room temperature; incubated the PVDF membranes at $4^{\circ} \mathrm{C}$ overnight respectively with antibody IL-10 (1:500), JAK2 (1:1000), STAT3 (1:2000) and $\beta$-actin (1:5000), rinsed with TBST $3 \times 10$ mins, incubate the PVDF membranes $1 \mathrm{~h}$ at room temperature respectively with rabbit fluorescent IgG (1:5000) and rat fluorescent IgG (1:2000), rinsed with TBST $3 \times 10$ mins, obtained the target protein bands by ECL chemiluminescence and film developing, Image $J$ software was used to analyze the grayscale values of each target band, using $\beta$-actin as the internal reference.

\subsection{Statistical Analysis}

The results are indicated by mean \pm standard deviation $(\bar{X} \pm s)$, One-way ANOVA followed by the Bonferroni method test was used to determine statistical

Table 2. Primer sequences of mRNA.

\begin{tabular}{|c|c|c|c|c|}
\hline mRNA & & Primer sequences & $\operatorname{Tm}\left({ }^{\circ} \mathrm{C}\right)$ & $\begin{array}{c}\text { Fragment } \\
\text { size (bp) }\end{array}$ \\
\hline \multirow{2}{*}{$\beta$-actin } & forward primer & 5'-GGAGATTACTGCCCTGGCTCCTA-3' & 64.9 & \multirow[b]{2}{*}{150} \\
\hline & reverse primer & 5'-GACTCATCGTACTCCTGCTTGCTG-3' & 64.7 & \\
\hline \multirow{2}{*}{ IL-10 } & forward primer & 5'-CAGACCCACATGCTCCGAGA-3' & 64.8 & \multirow{2}{*}{141} \\
\hline & reverse primer & 5'-CAAGGCTTGGCAACCCAAGTA-3' & 64.5 & \\
\hline \multirow{2}{*}{ STAT3 } & forward primer & 5'-TTTGAGACAGAGGTGTACCACCAAG-3' & 63.8 & \multirow{2}{*}{118} \\
\hline & reverse primer & 5'-ACCACAGGATTGATGCCCAAG-3' & 64.4 & \\
\hline \multirow{2}{*}{ JAK2 } & forward primer & 5'-TTTGAAGACAGGGACCCTACACAG-3' & 63.7 & \multirow{2}{*}{101} \\
\hline & reverse primer & 5'-TCATAGCGGCACATCTCCACA-3' & 65.0 & \\
\hline
\end{tabular}


significance, and differences were considered significant at $\mathrm{P}<0.05$.

\section{Results}

1) Model: On the first day, one death in NC group, on the 28th days, a rat died with convulsion in EP group. The rats in EP group and GE groups showed different levels of seizure, most of them achieved lighting standards on the 18th day, manifested in obvious nod, facial and limb convulsions, upright vertical tail or fall or even systemic tonic spasm. The seizure situation on the 28th day was as in Table 3.

2) Effects of gastrodin on IL-10, STAT3 and JAK2 mRNA expression (Table 4 and Figure 1).

Table 3. The seizure situation on the 28th day $(\bar{X} \pm s)$.

\begin{tabular}{cccccc}
\hline Group & $\mathrm{N}$ & Dead & Episode latency $(\mathrm{s})$ & Episode duration $(\mathrm{s})$ & Attack strength \\
\hline NC & 10 & 1 & 0 & 0 & 0 \\
EP & 10 & 1 & $56.2 \pm 23.3$ & $2223.3 \pm 253.2$ & $4.0 \pm 0.7$ \\
GE1 & 10 & 0 & $80.4 \pm 33.4$ & $1830.7 \pm 448.8$ & $2.9 \pm 1.2$ \\
GE1 & 10 & 0 & $115.0 \pm 35.3^{* *}$ & $1291.4 \pm 459.8^{* *}$ & $2.3 \pm 1.1^{*}$ \\
GE1 & 10 & 0 & $101.1 \pm 33.8^{*}$ & $1674.8 \pm 394.7^{*}$ & $2.8 \pm 1.0$
\end{tabular}

Note: ${ }^{*} \mathrm{P}<0.05 ;{ }^{*} \mathrm{P}<0.01$ vs. EP group.

Table 4. Expression of hippocampal IL-10, STAT3 and JAK2 mRNA ( $\bar{X} \pm s)$.

\begin{tabular}{cccc}
\hline relative quantity & IL-10 & STAT3 & JAK2 \\
\hline NC & $1.00 \pm 0.00$ & $1.00 \pm 0.00$ & $1.00 \pm 0.00$ \\
EP & $0.13 \pm 0.06^{*}$ & $3.96 \pm 1.30^{* *}$ & $2.23 \pm 0.74^{*}$ \\
GE1 & $0.51 \pm 0.35$ & $2.65 \pm 0.35$ & $1.22 \pm 0.32$ \\
GE2 & $1.25 \pm 0.60^{\# \#}$ & $1.25 \pm 0.49^{\# \#}$ & $0.71 \pm 0.39^{\# \#}$ \\
GE3 & $0.69 \pm 0.45$ & $1.59 \pm 0.25^{\# \#}$ & $1.24 \pm 0.60$ \\
\hline
\end{tabular}

Note: ${ }^{*} \mathrm{P}<0.05,{ }^{* *} \mathrm{P}<0.01$ vs. NC group; ${ }^{*} \mathrm{P}<0.05,{ }^{* *} \mathrm{P}<0.01$ vs. EP group.

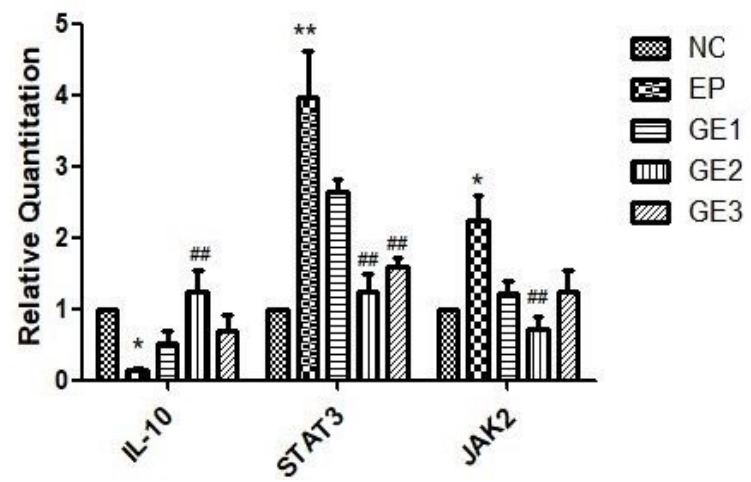

Figure 1. Gastrodin increased the expression of IL-10, and inhibited the expression of STAT3 and JAK2 in the hippocampus of the pentylenetetrazole rat model. Quantitative analysis by RT-qPCR $\left(\mathrm{n}=4 ;{ }^{\star} \mathrm{P}<0.05,{ }^{\star \star} \mathrm{P}<0.01\right.$ vs. NC; ${ }^{\#} \mathrm{P}<0.05,{ }^{\# \#} \mathrm{P}<0.01$ vs. EP). 
RT-qPCR showed that the expression of STAT3 and JAK2mRNA in the hippocampus of EP group was significantly higher than that of NC group $(\mathrm{P}<0.05)$, IL-10 mRNA was significantly decreased $(\mathrm{P}<0.05)$; IL-10 mRNA expression in GE2 group was significantly higher than that in EP group $(\mathrm{P}<0.01)$; compared with EP group, STAT3 mRNA expression in GE2 and GE3 group was decreased significantly $(\mathrm{P}<0.01)$, Jak2 mRNA in GE2 group was significantly lower than that in EP Group $(\mathrm{P}<0.05)$.

The mRNA levels of STAT3 and JAK2 higher while IL-10 was lower in the PTZ group than in the NC group. However the mRNA levels of STAT3 and JAK2 lower while IL-10 was higher in the GE2 group than in the PTZ group.

3) Effects of gastrodin on IL-10, STAT3 and JAK2 protein expression in hippocampus of rats (Table 5 and Figure 2).

Table 5. Expression of hippocampal IL-10, STAT3 and JAK2 protein ( $\bar{X} \pm s)$.

\begin{tabular}{cccc}
\hline Relative quantity & IL-10 & STAT3 & JAK2 \\
\hline NC & $1.03 \pm 0.02$ & $1.03 \pm 0.01$ & $1.05 \pm 0.03$ \\
EP & $0.45 \pm 0.23^{\star}$ & $5.94 \pm 3.31^{\star *}$ & $1.86 \pm 0.34^{\star}$ \\
GE1 & $0.54 \pm 0.16$ & $2.54 \pm 1.53$ & $1.11 \pm 0.19$ \\
GE2 & $1.06 \pm 0.41^{\#}$ & $1.81 \pm 0.83^{\#}$ & $0.72 \pm 0.19^{\# \#}$ \\
GE3 & $0.83 \pm 0.22$ & $1.97 \pm 0.57^{\#}$ & $1.00 \pm 0.58^{\#}$ \\
\hline
\end{tabular}

Note: ${ }^{\star} \mathrm{P}<0.05,{ }^{* \star} \mathrm{P}<0.01$ vs. NC group; ${ }^{\sharp} \mathrm{P}<0.01,{ }^{\# \#} \mathrm{P}<0.01$ vs. EP group.
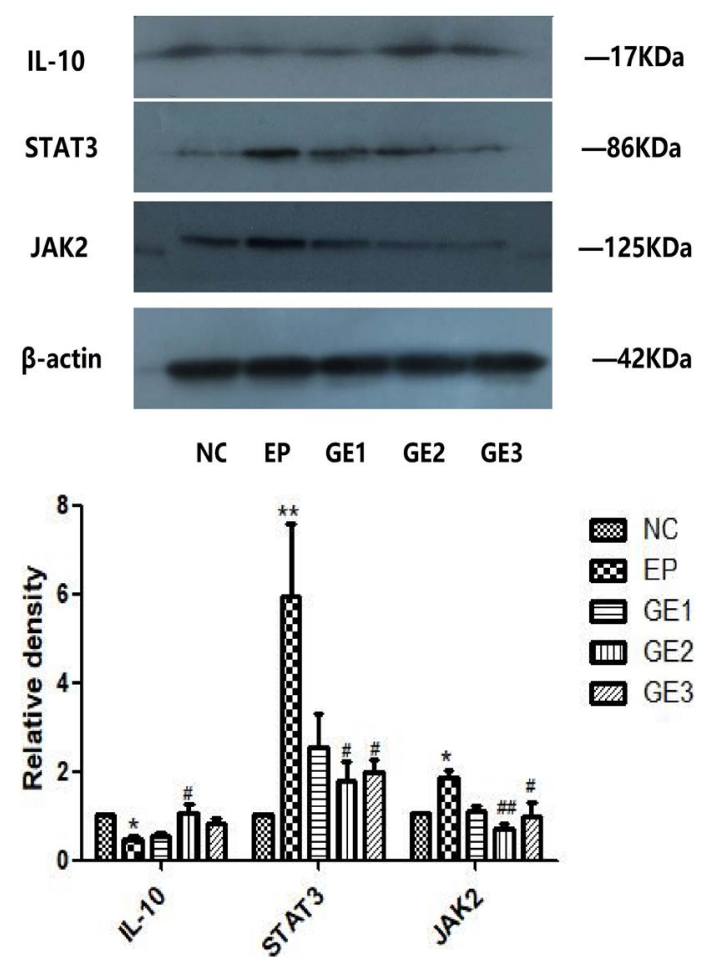

Figure 2. Gastrodin increased the expression of IL-10, and inhibited the expression of STAT3 and JAK2 in the hippocampus of the pentylenetetrazole rat model. (a) Western blots and (b) quantitative analysis of bands in $\mathrm{A}\left(\mathrm{n}=4 ;{ }^{*} \mathrm{P}<0.05,{ }^{* *} \mathrm{P}<0.01\right.$ vs. $\mathrm{NC} ;{ }^{*} \mathrm{P}<0.05,{ }^{\# *} \mathrm{P}<0.01$ vs. $\left.\mathrm{EP}\right)$. 
Compared with the NC group, the expression of IL-10 protein in EP group was significantly reduced $(\mathrm{P}<0.05)$, STAT3 increased significantly $(\mathrm{P}<0.01)$ and JAK2 increased significantly $(\mathrm{P}<0.05)$. Compared with the EP group, IL-10 protein expression in GE2 group was significantly higher $(\mathrm{P}<0.05)$, STAT3 decreased significantly $(P<0.05)$ and JAK2 decreased significantly $(P<0.01)$, STAT3 and JAK2 in GE3 group decreased significantly $(\mathrm{P}<0.05)$.

The protein levels of STAT3 and JAK2 were higher while IL-10 was lower in the PTZ group than in the NC group. However, the protein levels of STAT3 and JAK2 were lower while IL-10 was higher in the GE2 group than in the PTZ group.

\section{Discussions}

The pathogenesis of epilepsy is complex, involving neuronal electrophysiological activities, mainly attributed to the imbalance of excitatory and inhibition of the central nervous system, and is closely related to the neurotransmitter imbalance, ion channel, glial cell, heredity, inflammatory reaction and immune abnormality. In clinical, for the traditional single target of antiepileptic drugs mainly in western medicine which has larger side effects, patients can not adhere to long-term use, and they failed to obtain satisfactory curative effect, thus exploring multi-channel, multi-target, effective and low toxic antiepileptic drugs, has become the inevitable way to treat epilepsy.

In our long-term clinical work, we found that for conventional western medicine difficult to control epilepsy (especially the persistent state of epilepsy), combined with gastrodin injection, seizures could be better controlled. Gastrodin is the dry tuber of gastrodia elata, which has anti-inflammatory, scavenging free radicals and anti-apoptotic effects. In the early experiments, we found that gastrodin could promote the expression of cytokines IL-10, inhibit IL-1 $\beta$ and TNF- $\alpha$ in hippocampal neurons, exert an antiepileptic effect by inhibiting the inflammatory reaction in hippocampus of epileptic rats [8]. On the other hand, the JAK/STAT pathway is a key signaling pathway for central nervous system mediated inflammation and immune response [11]. Therefore, this experiment is based on the inflammatory cytokine IL-10 and JAK2/STAT3 as the target, to investigate the molecular target of antiepileptic action of gastrodin through IL-10 regulating inflammatory signaling pathway.

From the perspective of cytokines, in this experiment, compared with NC group, the expression of IL-10 in the hippocampus of EP group was significantly decreased, and it was significantly increased after the intervention of medium dose gastrodin $(6 \mathrm{mg} / \mathrm{kg})$. An important role of IL-10 is to inhibit the secretion of inflammatory factors such as IL-1, IL-6, IL-12 and tumor necrosis factor in mast cells and macrophages [12]. In addition, some studies have shown that IL-10 has a significant inhibitory effect on the secretion of TNF- $\alpha$ and IL-2 in peripheral blood mononuclear cells of epileptic rats, and the inhibitory effect was dependent on concentration [13]. In epilepsy mice model induced by pilocarpine, the expression of IL-10 decreased, IL-1 $\beta$, IL-6, TNF- $\alpha$ increased in the 
hippocampus of the mice, and the intervention of swertia glucoside $(450 \mathrm{mg} / \mathrm{kg})$ could significantly increase the expression of IL-10, and reduce the expression of IL- $1 \beta$, IL-6, TNF- $\alpha$ [14]. In a meta-analysis of inflammatory mediators in epilepsy patients, it was found that the levels of IL-10 in serum and cerebrospinal fluid in epileptic patients increased, and IL-10 improved immune pathology by preventing inflammation-related tissue damage, which played a protective role in the development of epilepsy [3]. The above studies indicate that taking IL-10 as the target point may play an active role in the treatment of epilepsy. At present, many scholars have started to study the polymorphism of IL-10 promoter gene, to study the changes of IL-10 related genotypes after drug intervention, and to find the indexes of evaluating drug efficacy [15].

From the perspective of JAK2/STAT3 target, our experimental results show that the JAK2 and STAT3 expression in EP Group is significantly higher than that in NC group, and the JAK2 and STAT3 positive expression are significantly decreased after the intervention of gastrodin. The secretion of anti-inflammatory cytokines IL-10 decreased during epileptic seizures, and the inflammatory cytokines such as IL-1, IL-6, IL-12 and TNF increased, and the glial cells have many targets of cytokines (such as IL-1, IL-6, TNF- $\alpha$ ). When these cytokines are combined with tyrosine kinase-related receptors on glial cells, the gp130-mediated JAK/STAT signal transduction pathway is activated, and STAT is activated by phosphorylation to form dimer, expose to the access nuclear signals, transferred to the nucleus and regulate the expression of the target gene, leading to the proliferation of astrocytes [16]. JAK/STAT signal pathway plays a vital role in the differentiation and proliferation of astrocytes, and the proliferation of astrocytes is an important pathological change in the brain tissue of epileptic patients. In the temporal lobe epilepsy rat model, the JAK2/STAT3 signaling pathway is activated, and the using JAK2/STAT3 specific inhibitor WP 1066 can reduce the frequency and severity of the occurrence of epilepsy [17]. Another study showed that the specific blocking JAK2/STAT3 pathway could significantly inhibited the expression of synaptic protein in temporal lobe epilepsy model rats' hippocampus, regulating the hippocampal synaptic plasticity and played an important antiepileptic role [18]. Therefore, the occurrence of epilepsy is closely related to IL-10 and JAK2/STAT3. In our study, compared with NC group the expression of IL-10 in EP group was significantly decreased, JAK2 and STAT3 significantly increased, and the expression of IL-10 increased, JAK2, STAT3 expression decreased obviously after the intervention of medium dose gastrodin $(6 \mathrm{mg} / \mathrm{kg})$, This indicate that gastrodin can exert antiepileptic effect by improving IL-10 expression and inhibiting JAK2, STAT3 expression.

Above all, gastrodin have an antiepileptic effect on PTZ-induced epileptic rats, its mechanism may be related to increase hippocampal tissue IL-10 expression, inhibit JAK2, STAT3 expression, thus regulating the JAK2/STAT3 pathway. However, there are many signaling pathways involved in the development of epilepsy, and it is necessary to further verify the antiepileptic effects of gastrodin 
via IL-10 whether relate to other signaling pathways.

\section{Acknowledgements}

This study was funded by Hubei Province health and family planning commission science and technology project in 2012 (JX6B33, Xianglin Cheng). Thanks the experimental technical help by all teachers in the first affiliated hospital central laboratory of Yangtze University.

\section{References}

[1] Liu, M. and Xie, P. (2014) Neurology. People's Medical Publishing House, Beijing, 167.

[2] Legido, A. and Katsetos, C.D. (2014) Experimental Studies in Epilepsy: Immunologic and Inflammatory Mechanisms. Seminars in Pediatric Neurology, 21, 197-206. https://doi.org/10.1016/j.spen.2014.10.001

[3] de Vries, E.E., Van, D.M.B., Braun, K.P., Van, R.A., De, J.W. and Jansen, F.E. (2016) Inflammatory Mediators in Human Epilepsy: A Systematic Review and $\mathrm{Me}$ ta-Analysis. Neuroscience \& Biobehavioral Reviews, 63, 177-190. https://doi.org/10.1016/j.neubiorev.2016.02.007

[4] Engelhardt, K.R. and Grimbacher, B. (2014) Il-10 in Humans: Lessons from the Gut, il-10/il-10 Receptor Deficiencies, and il-10 Polymorphisms. Current Topics in Microbiology \& Immunology, 380, 1. https://doi.org/10.1007/978-3-662-43492-5_1

[5] Dennis, K.L., Blatner, N.R., Gounari, F. and Khazaie, K. (2013) Current Status of Interleukin-10 and Regulatory t-Cells in Cancer. Current Opinion in Oncology, 25, 637. https://doi.org/10.1097/CCO.0000000000000006

[6] Saraiva, M. and O'Garra, A. (2010) The Regulation of IL-10 Production by Immune Cells. Nature Reviews Immunology, 10, 170. https://doi.org/10.1038/nri2711

[7] Kwilasz, A.J., Grace, P.M., Serbedzija, P., Maier, S.F. and Watkins, L.R. (2014) The Therapeutic Potential of Interleukin-10 in Neuroimmune Diseases. Neuropharmacology, 96, 55-69.

[8] Li, F., Sun, Y.L., Cheng, X.L., Cai, G.X., Cai, X.Y., Xu, X.M., et al. (2015) The Antiepileptic Mechanism of Gastrodin on the Hippocampal Inflammatory Signaling Pathway in Epileptic Rats. Practical Medicine and Clinic, No. 5, 510-512.

[9] Racine, R.J., Steingart, M. and Mcintyre, D.C. (1999) Development of Kindling-Prone and Kindling-Resistant Rats: Selective Breeding and Electrophysiological Studies. Epilepsy Research, 35, 183-195. https://doi.org/10.1016/S0920-1211(99)00013-3

[10] Livak, K.J. and Schmittgen, T.D. (2001) Analysis of Relative Gene Expression Data Using Real-Time Quantitative PCR and the 2(-Delta Delta $\mathrm{c}(\mathrm{t})$ ) Method. Methods, 25, 402-408. https://doi.org/10.1006/meth.2001.1262

[11] Nicolas, C.S., Mascia, A., Bortolotto, Z.A., Andrew, D., Zsolt, C., Assia, F., et al. (2013) The Role of JAK-STAT Signaling within the CNS. JAK-STAT, 2, e22925. https://doi.org/10.4161/jkst.22925

[12] Zhou, L., Zhou, G.Y. and Lu, L.M. (2012) The Bidirectional Immune Regulation of IL-10. Chinese Journal of Cellular and Molecular Immunology, 28, 1100-1102.

[13] Fei, M. (2006) The Experimental Study on the Secretion of IL-2 and TNF- $\alpha$ in the Peripheral Blood of Epileptic Rats. Doctoral Dissertation, Shanxi Medical University, Taiyuan. 
[14] Deng, X.H., Zhang, X., Wang, J., Ma, P.S., Ma, L. and Niu, Y., et al. (2017) Anticonvulsant Effect of Swertiamarin against Pilocarpine-Induced Seizures in Adult Male Mice. Neurochemical Research, 42, 3103-3113. https://doi.org/10.1007/s11064-017-2347-0

[15] Ying, B., Shi, Y., Pan, X., Song, X., Huang, Z. and Niu, Q., et al. (2011) Association of Polymorphisms in the Human Il-10 and Il-18 Genes with Rheumatoid Arthritis. Molecular Biology Reports, 38, 379-385. https://doi.org/10.1007/s11033-010-0119-x

[16] Hebert, M.A. and O’Callaghan, J.P. (2000) Protein Phosphorylation Cascades Associated with Methamphetamine-Induced Glial Activation. Annals of the New York Academy of Sciences, 914, 238-262. https://doi.org/10.1111/j.1749-6632.2000.tb05200.x

[17] Grabenstatter, H.L., Angel, Y.C.D., Carlsen, J., Wempe, M.F., White, A.M. and Cogswell, M., et al. (2014) The Effect of STAT3 Inhibition on Status Epilepticus and Subsequent Spontaneous Seizures in the Pilocarpine Model of Acquired Epilepsy. Neurobiology of Disease, 62, 73-85. https://doi.org/10.1016/j.nbd.2013.09.003

[18] Gu, J.H., Sun, T., Li, X.G., Xia, H.C. and Wang, F. (2011) The Regulatory Role of JAK/STAT3 Pathway for the Synaptic Plasticity of Epileptic Rat Temporal Lobe. Academic Conference of the Ninth National Youth Physiological Workers of China, Beijing, 21 October 2011, 2. 